\title{
Effects of CDK6 regulated by miR-298 on proliferation and apoptosis of thyroid cancer cells
}

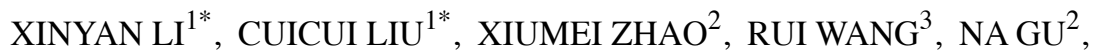 \\ HONGSHENG SHEN ${ }^{2}$, XIJING LI ${ }^{2}$, LUYAO WANG ${ }^{2}$ and CHAO LI ${ }^{1}$ \\ ${ }^{1}$ Department of Pharmacology, Cangzhou Medical College, Cangzhou, Hebei 061000; \\ ${ }^{2}$ Centre for Research and Development of Anti-tumor Drugs, Tianjin Institute of Medical \\ and Pharmaceutical Sciences, Tianjin 300020; ${ }^{3}$ Department of Critical Care Medicine, \\ Cangzhou Central Hospital, Cangzhou, Hebei 061001, P.R. China
}

Received November 15, 2019; Accepted January 10, 2020

DOI: $10.3892 / 01.2020 .11398$

\begin{abstract}
Effects of CDK6 regulated by miR-298 on proliferation and apoptosis of thyroid cancer cells were explored. Seventy-five cases of thyroid carcinoma and adjacent tissues were collected. The expression levels of miR-298 and CDK6 mRNA in tissues and cells were detected by RT-PCR. In addition, thyroid cancer cells and human normal thyroid cells Nthy-ori3-1 were purchased, with the former transfected with miR-298-mimics, miR-298-inhibitor, miR-NC, si-CDK6, si-NC, Sh-CDK6, Sh-NC to build cell models. Then the expression levels of miR-298 and CDK6 in thyroid cancer tissues and cells were detected by qRT-PCR, and the expression of CDK6, Bax, Bcl- 2 and caspase- 3 by WB. CCK- 8 and flow cytometry were employed to detect cell proliferation and apoptosis, and dual luciferase report was adopted to determine the relationship between miR-298 and CDK6. miR-298 was underexpressed in thyroid cancer, and CDK6 was highly expressed in thyroid cancer. Cell experiments revealed that overexpression of miR-298 or inhibition of CDK6 expression could suppress cell proliferation, promote apoptosis, and significantly increase the expression levels of Bax and caspase-3 proteins, decrease Bcl-2 protein expression, which was contrary to the biological phenotype of cells after inhibition of miR-298 or further overexpression of CDK6. Dual luciferase report confirmed that miR-298 was a targeting site of CDK6. miR-298 can inhibit the proliferation of thyroid cells and promote apoptosis of thyroid cancer cells by regulating the
\end{abstract}

Correspondence to: Dr Cuicui Liu, Department of Pharmacology, Cangzhou Medical College, 1 Guangzhou Road, Canal Area, Cangzhou, Hebei 061000, P.R. China

E-mail: cc97e2@163.com

*Contributed equally

Key words: miR-298, CDK6, thyroid cancer, proliferation, apoptosis, influence expression of CDK6, which is expected to be a potential target for clinical application.

\section{Introduction}

Thyroid cancer is a common malignant tumor of the endocrine system, its incidence keeps increasing year by year driven by the change of social environment (1). However, due to the latent onset of thyroid cancer, many patients are diagnosed with metastasis, hazarding the prognosis of patients $(2,3)$. The fact that the current diagnosis and treatment of thyroid cancer offer few options results in unsatisfactory therapeutic effect for many patients (4). Therefore, exploring the pathological mechanism of thyroid cancer is of great clinical significance for the diagnosis and treatment of patients with thyroid cancer. miRNAs are non-coding microRNAs, which mainly influence the biological function of cells through mRNA matching with downstream target genes (5). Studies have shown that miRNAs play a vital part in the occurrence and development of thyroid cancer. For example, miR-26a-5p has been reported to inhibit proliferation, invasion and migration of thyroid papillary cancer cells by inhibiting expression of Wnt5a (6). According to some other studies (7), miR-15a can affect the proliferation and apoptosis of thyroid cancer cells by regulating AKT. Among those miRNAs, miR-298, located on human chromosome $20 \mathrm{q} 13.32$, is related to the proliferation and invasion of tumor cells according to recent studies (8). For example, a study found that miR-298 could affect the proliferation and invasion of ovarian cancer cells by regulating the expression of EZH2 (9). However, the role and mechanism of miR-298 in thyroid cancer remains a subject of investigation. While CDK6 is a kinase-catalyzed group of a protein kinase complex that primarily affects the cell cycle, which can increase cell proliferation by accelerating cell cycle (10). However, similarly to miR-298 little research has been conducted on the effect of CDK6 on thyroid cancer cells.

Both Targetscan and miRDB databases predict that CDK6 is a target gene of miR-298, so it was speculated that miR-298 could affect thyroid cancer cells by regulating CDK6. Therefore, thyroid cancer cells were selected as the 
research subjects in the present study to evaluate the effect and mechanism of miR-298 on thyroid cancer cells, in an attempt to provide a new target direction for the research on thyroid cancer.

\section{Patients and methods}

Clinical specimens. Seventy-five patients who underwent thyroidectomy in Cangzhou Medical College (Cangzhou, China) from January 2016 to January 2018 were enrolled. Paired thyroid cancer tissues and adjacent cancer tissues were obtained from each patients during the operation, and stored in a liquid nitrogen tank. The patient information is detailed in Table I. Inclusion criteria: Patients pathologically diagnosed as thyroid cancer for the first time were included. In contrast, the exclusion criteria were as follows: Patients who had received chemoradiotherapy, associated with other malignant tumors, severe liver or kidney dysfunction, severe infectious diseases, or those refused to provide experimental specimens were excluded.

The study was approved by the Ethics Committee of Cangzhou Medical College (Cangzhou, China). Patients who participated in this research had complete clinical data. Patients and their families agreed to participate in the experiment and signed informed consents were obtained from the patients or the guardians.

Experimental reagents and materials. Human thyroid cancer lines SW579, KHM-2, B-CPAP and human normal thyroid cell line Nthy-ori3-1 (Conservation Genetics CAS Shanghai Cell Bank, China). QRT-PCR and reverse transcription kit (AQ201-01, AQ202-01; TransGen Biotech Co., Ltd.), PBS, fetal bovine serum (FBS) (10010049 and 10437028; Gibco; Thermo Fisher Scientific, Inc.), TRIzol reagent (15596018; Gibco; Thermo Fisher Scientific, Inc.), dual luciferase reporter detection kit (KFS303-TFX; Beijing Biolab Technology Co., Ltd.), CCK-8 kit (Promega Corporation), transwell kit (Beijing Yaanda Biotechnology Co., Ltd.), RIPA, BCA protein kit (Gibco; Thermo Fisher Scientific, Inc.), Annexin V-FITC/PI cell apoptosis kit (Zp327-1; Beijing Zoman Biotechnology Co., Ltd.), CDK6, caspase-3, Bax, Bcl-2 and $\beta$-actin antibodies (Cell Signaling Technology Co.), goat anti-rabbit IgG secondary antibody (Wuhan Boster Biological Technology Co., Ltd.), ECL developer (Gibco; Thermo Fisher Scientific, Inc.), PCR instrument (7500 real-time PCR system; Applied Biosystems; Thermo Fisher Scientific, Inc.). All primers were designed and synthesized by Shanghai Sangon Biotechnology Co., Ltd.

RT-PCR detection for miR-298 and CDK6 expression. Thyroid tissues and adjacent tissues were removed from the liquid nitrogen tank for grinding. The total RNA in the tissue was extracted with TRIzol reagent, whose concentration and purity of total RNA were then detected by ultraviolet spectrophotometer, and those within OD260/OD280 >1.8 were selected for further experiments. Then, $5 \mu \mathrm{g}$ of total RNA was taken for cDNA reverse transcription according to the kit instructions. The reaction parameters were: $37^{\circ} \mathrm{C}$ for $15 \mathrm{~min}$, $42^{\circ} \mathrm{C}$ for $35 \mathrm{~min}$, and $70^{\circ} \mathrm{C}$ for $5 \mathrm{~min}$. miR-298 amplification conditions: PCR reaction conditions: Pre-denaturation at $94^{\circ} \mathrm{C}$ for $45 \mathrm{sec}$, denaturation at $94^{\circ} \mathrm{C}$ for $10 \mathrm{sec}$, annealing at $60^{\circ} \mathrm{C}$ for $45 \mathrm{sec}$, totaling 40 cycles. CDK6 amplification conditions: Pre-denaturation at $95^{\circ} \mathrm{C}$ for $30 \mathrm{sec}$, denaturation at $95^{\circ} \mathrm{C}$ for $10 \mathrm{sec}$, annealing at $60^{\circ} \mathrm{C}$ for $35 \mathrm{sec}$, and a total of 40 cycles were performed. Three replicate wells were set per sample, and the experiment was carried out 3 times. Finally, with U6 as the internal reference of miR-298, and $\beta$-actin for CDK6, $2^{-\Delta \Delta c t}$ was applied to analyze the data.

Cell culture and transfection. Thyroid cancer cell lines were cultured in a medium containing $10 \%$ PBS DMEM at $37^{\circ} \mathrm{C}$ and $5 \% \mathrm{CO}_{2}$. When the cell adherent growth and fusion reached $85 \%, 25 \%$ trypsin was added for digestion before further culture and passage in the culture medium. After passage, the cells were taken for detection of the expression levels of miR-298 and CDK6 mRNA by the method described. Expression of miR-298 in SW579 and KHM-2 cells was lower than that in B-CPAP cells, thus, SW579 and KHM-2 cells were selected for transfection and subsequent experiments. miR-298-mimics (overexpression sequence), miR-298-inhibitor (inhibition sequence), miR negative control (miR-NC), targeted inhibition of CDK6 RNA (si-CDK6), negative control RNA (Si-NC), Targeting overexpressing CDK6 RNA (sh-CDK6), negative control (Sh-NC) were transfected into cells using Lipofectamine ${ }^{\mathrm{TM}} 2000$ kit, strictly following the kit instructions.

Western blot analysis. RIPA lysis was applied to lyse the cells and extract total protein, then the protein concentration was measured by BCA assay. The protein concentration was adjusted to $4 \mu \mathrm{g} / \mu 1$, electrophoretically separated by $12 \%$ SDS-PAGE before transferring to PVDF membrane, then sealed for $2 \mathrm{~h}$ with 5\% skim milk powder. CDK6 (1:500), caspase-3 (1:500), Bax (1:500), Bcl-2 (1:500) and $\beta$-actin $(1: 1,000)$ were then added and sealed overnight at $4^{\circ} \mathrm{C}$. After that, the first antibody was removed by washing the membrane, followed by the addition of horseradish peroxidase-labeled goat anti-rabbit secondary antibody $(1: 1,000)$, incubated at $37^{\circ} \mathrm{C}$ for $1 \mathrm{~h}$, and rinsed with PBS 3 times, 5 min each. Then, developed in a dark room, excess liquid was dried on the membrane with filter paper, then developed with ECL luminescence.

Cell proliferation test. The proliferation ability of SW579 and KHM-2 cells was evaluated by CCK- 8 kit. Cells were collected $48 \mathrm{~h}$ after transfection, adjusted to $3 \times 10^{4}$ cells and inoculated on 96-well plates. Then, $100 \mu \mathrm{l}$ cells were implanted in each well and cultured in an environment of $37^{\circ} \mathrm{C}$ and $5 \% \mathrm{CO}_{2}$. Next, each well was added with $10 \mu \mathrm{l}$ CCK8 solution at $0,24,48$, and $72 \mathrm{~h}$ after cell adherent growth. After adding the reagent, the culture was continued for $2 \mathrm{~h}$ in an incubator at $37^{\circ} \mathrm{C}$ and $5 \% \mathrm{CO}_{2}$ for $2 \mathrm{~h}$. Then the OD value was measured at $450 \mathrm{~nm}$ with a microplate reader to detect cell proliferation and draw a growth curve. The experiment was repeated 3 times.

Apoptosis. The transfected cells were digested with $0.25 \%$ trypsin, washed twice with PBS, and added with $100 \mu \mathrm{l}$ binding buffer to prepare a suspension of $1 \times 10^{6}$ cells $/ \mathrm{ml}$. Followed by successive addition of Annexin V-FITC and PI, incubated at room temperature for $5 \mathrm{~min}$ in the dark, and then detected by FACSVerse flow cytometry system. The experiment was repeated 3 times for average value. 

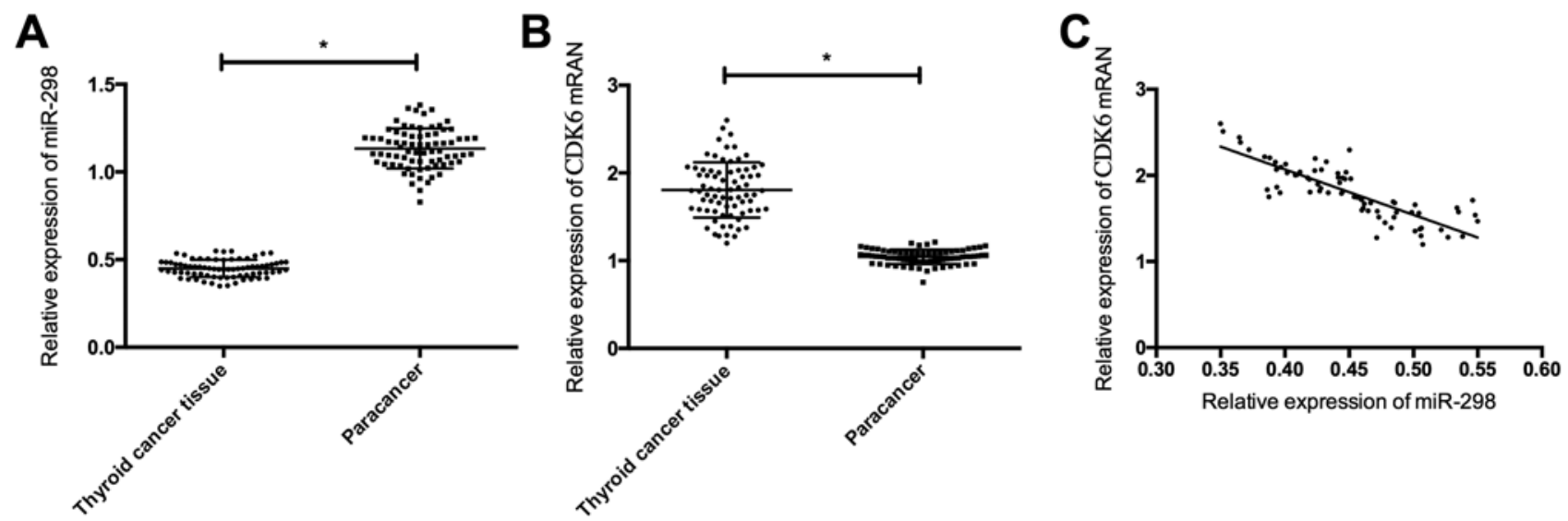

Figure 1. Expression levels of miR-298 and CDK6 in thyroid carcinoma tissues. (A) Expression of miR-298 in thyroid carcinoma. (B) Expression of CDK6 in thyroid carcinoma. (C) Correlation analysis of miR-298 and CDK6 expression levels. " $\mathrm{P}<0.05$.

Table I. General information of patients.

\begin{tabular}{ll}
\hline Categories & Thyroid cancer patients $(\mathrm{n}=75)$ \\
\hline Sex & $39(52.00)$ \\
Male & $36(48.00)$ \\
Female & $58.25 \pm 8.92$ \\
Age (years) & $22.35 \pm 1.12$ \\
BMI (kg/m $\left.{ }^{2}\right)$ & \\
Pathological types & $31(41.33)$ \\
Papillary carcinoma & $15(20.00)$ \\
Follicular carcinoma & $17(22.67)$ \\
Undifferentiated carcinoma & $12(16.00)$ \\
Medullary carcinoma & \\
Pathological stage & $32(42.67)$ \\
I & $27(36.00)$ \\
II & $16(21.33)$ \\
III & \\
Differentiation degree & $33(44.00)$ \\
High & $26(34.67)$ \\
Medium & $16(21.33)$ \\
Low &
\end{tabular}

Dual luciferase assay. Lipofectamine 2000 kit was employed to transfect CDK6-3'UTR wild-type (Wt), CDK6-3'UTR mutant (Mut) as well as miR-298-mimics and miR-NC into SW579 and KHM-2 cells. Luciferase activity was measured $48 \mathrm{~h}$ after transfection using a dual luciferase reporter assay kit (Promega Corporation).

Statistical analysis. In the present study, the collected data was analysed using SPSS20.0 (IBM Corp.), and the picture rendering was performed by GraphPad 7. Independent t-test was employed for inter-group comparison, while the inter-group comparison was conducted by one-way ANOVA. LSD- $t$ was adopted for post pairwise comparison, and repeated measurement ANOVA was applied for multi-time expression. Bonferroni was used for post hoc test, and Pearson test was utilized to analyze the correlation between micR-298 and CDK6 in tissues. $\mathrm{P}<0.05$ indicates a statistically significant difference.

\section{Results}

Expression levels of $m i R-298$ and CDK6 in thyroid carcinoma tissues. RT-PCR showed that compared with the adjacent tissues, miR-298 decreased notably $(\mathrm{P}<0.05)$, and CDK6 increased significantly in thyroid cancer tissues $(\mathrm{P}<0.05)$. There was a negative correlation between the expression levels of miR-298 and CDK6 ( $\mathrm{r}=-0.845, \mathrm{P}<0.05$ ) (Fig. 1).

Effects of miR-298 on proliferation and apoptosis of thyroid cancer cells. The detection of expression levels of miR-298 in SW579, KHM-2, B-CPAP and human normal thyroid cells Nthy-ori3-1 revealed that the miR-298 expression in thyroid cancer cells SW579, KHM-2, B-CPAP were significantly lower than that in Nthy-ori3-1 cells. miR-298 expression in SW579 and KHM-2 cells transfected with miR-298-mimics was significantly higher than that in cells transfected with miR-NC, while the miR-298 expression in miR-298-inhibitor cells was significantly decreased. The biological functions of the cells in the two groups showed that, compared with the miR-NC group, the proliferation of transfected miR-298-mimics was remarkably decreased, the apoptosis rate was significantly increased, while the proliferation of transfected miR-298-inhibitor cells was significantly enhanced, and the apoptosis rate was significantly decreased. The transfected miR-298-mimics cells presented markedly reduced Bcl-2 expression and significantly increased expression levels of caspase-3 and Bax protein in contrast with the miR-NC group, while the opposite effect was observed between transfected miR-298-inhibitor cells and the miR-NC group (Fig. 2).

Effects of CDK6 on the biological function of thyroid cancer cells. Detection of expression levels of CDK6 in SW579, KHM-2, B-CPAP and the human normal thyroid cells Nthy-ori3-1 revealed that the CDK6 expression in thyroid cancer cells SW579, KHM-2 and B-CPAP were significantly higher than that in Nthy-ori3-1 cells. Compared with si-nc transfected cells, the expression of CDK6 in SW579 and KHM-2 cells transfected with Si-CDK6 was significantly 


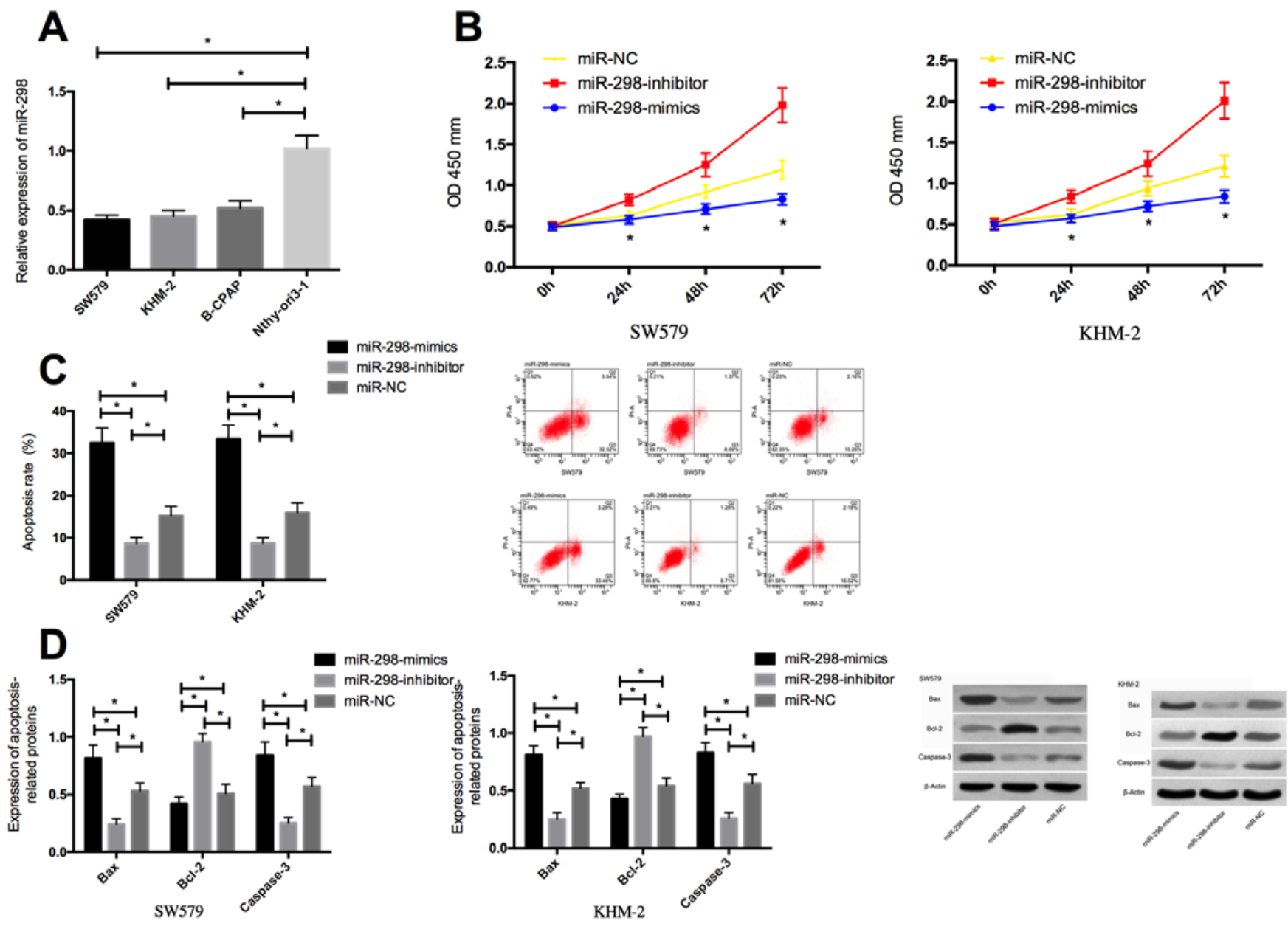

Figure 2. Effects of miR-298 on proliferation and apoptosis of thyroid cancer cells. (A) Expression of miR-298 in thyroid cancer cells. (B) Effects of miR-298 on proliferation of SW579 and KHM-2 cells. (C) Effects of miR-298 on apoptosis rate of SW579 and KHM-2 cells. (D) Effects of miR-298 on apoptosis-related proteins of SW579 and KHM-2 cells. ${ }^{*} \mathrm{P}<0.05$.

downregulated, while the expression of CDK6 in Sh-CDK6 transfected cells was markedly declined. The biological functions of the cells in the two groups indicated that, compared with the Si-NC group, the proliferation of transfected Si-CDK6 cells was remarkably decreased, apoptosis was significantly increased, the expression of $\mathrm{Bcl}-2$ was markedly reduced, and the expression levels of caspase- 3 and Bax protein were significantly enhanced. While the proliferation of transfected Sh-CDK6 cells was significantly enhanced, the apoptosis rate was notably decreased, the expression of Bcl-2 protein was significantly increased, and the expression of caspase- 3 and Bax protein was significantly decreased in contrast to cells in the Si-NC group (Fig. 3).

Identification of miR-298 target genes. To further validate the relationship between miR-298 and CDK6, we first predicted the presence of targeted binding sites between CDK6 and miR-298 by predicting the target gene downstream of miR-298 by Targetscan 7.2. Then dual luciferase activity was performed to verify that prediction. The results showed that the luciferase activity of CDK6-3'UT Wt was markedly decreased after miR-298 overexpression $(\mathrm{P}<0.05)$, but had no effect on that of CDK6-3'UTR Mut (P>0.05). WB detection indicated that CDK6 protein expression of SW579 and KHM-2 cells was significantly decreased after transfection with miR-298-mimics, while it was significantly increased after transfection with miR-298-inhibitor $(\mathrm{P}<0.05)$ (Fig. 4).

\section{Discussion}

As a common malignant tumor of the endocrine system, thyroid cancer presents a relatively intricate pathogenesis (11). In recent years, the role of miRNA in thyroid cancer has been gradually recognized, and many studies have reported that miRNAs can regulate the biological function of thyroid cancer cells $(12,13)$.

In our study, miR-298 was found to be significantly downregulated in thyroid cancer tissues, suggesting that miR-298 may be associated with the development of thyroid cancer. The expression of miR-298 in thyroid cancer cells was further tested and a consistent conclusionwas reached. Studies in the past demonstrated that miR-298 exerted oncogene function in some malignant tumors, for example, it could inhibit the progress of hepatocellular carcinoma by inhibiting expression of CTNND1 (14). In order to confirm our hypothesis that miR-298 also played a role in tumor suppressor genes in thyroid cancer, we subsequently overexpressed and underexpressed miR-298 in thyroid 
A

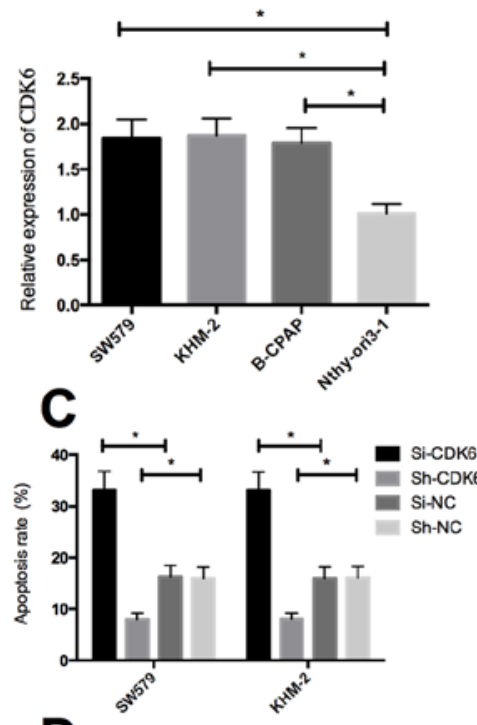

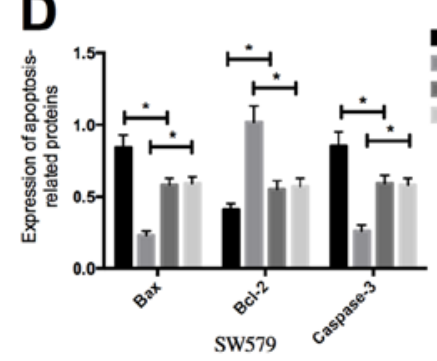

B

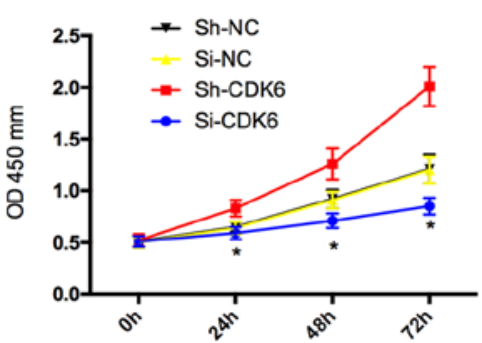

SW579

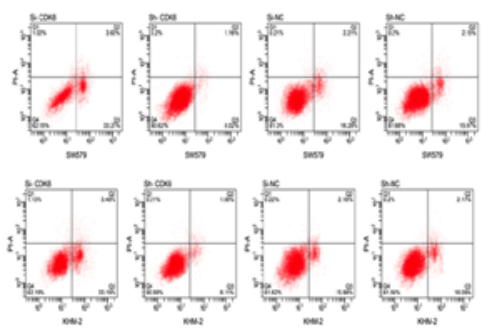$$
\text { (1) }
$$

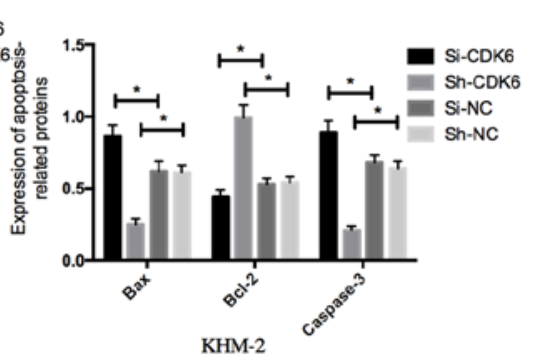

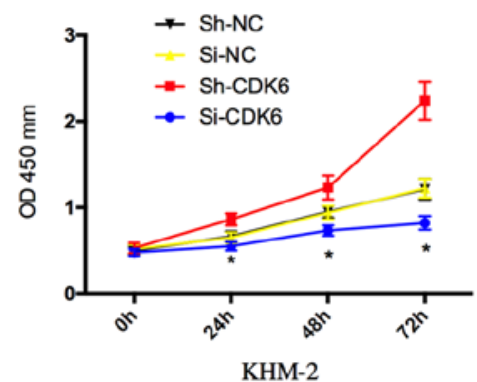

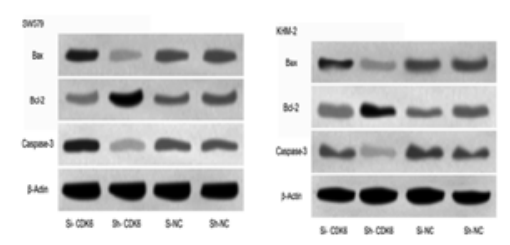

Figure 3. Effects of CDK6 on proliferation and apoptosis of thyroid cancer cells. (A) Expression of CDK6 in thyroid cancer cells. (B) Effects of CDK6 on proliferation of SW579 and KHM-2 cells. (C) Effects of CDK6 on apoptosis of SW579 and KHM-2 cells. (D) Effects of CDK6 on apoptosis-related proteins in SW579 and KHM-2 cells. ${ }^{*} \mathrm{P}<0.05$.

A

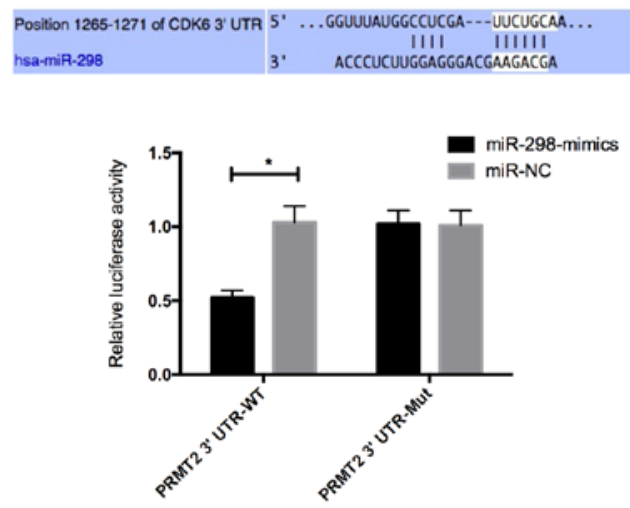

B
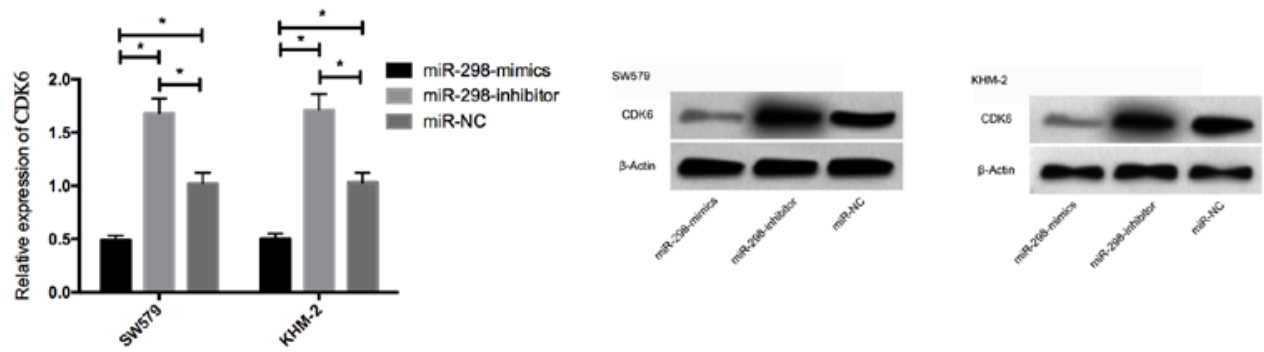

Figure 4. Identification of miR-298 target genes. (A) There were binding sites between miR-298 and CDK6, relative luciferase activity - dual luciferase reporter assay. (B) Effects of miR-298 on CDK6 protein expression in thyroid cancer cells. " $\mathrm{P}<0.05$. 
cancer cells SW579 and KHM-2 respectively. The results showed that after the overexpression of miR-298, the proliferation of SW579 and KHM-2 cells was significantly inhibited, the apoptosis rate was significantly increased, and the apoptosis-related protein was also consistent with the apoptosis rate. Vice versa, the underexpressed miR-298 brought about significantly enhanced proliferation ability, and notably reduced apoptosis rate of SW579 and KHM-2 cells, suggesting that miR-298 also plays a role of oncogene in thyroid cancer, which was consistent with the role of miR-298 in other tumors. In turn, it also suggested that the low expression of miR-298 might be one of the causes of thyroid cancer.

However, the mechanism of miR-298 in thyroid cancer remains poorly understood. Generally speaking, miRNAs regulate tumor cells by acting on their target genes (15), and we found a targeted relationship between miR-298 and CDK6 through Targetscan and miRDB database analysis. CDK6, as a kinase-catalyzed subunit, has been suggested by previous studies that the overexpression or activation of CDK6 is closely related to the occurrence of many malignant tumors, such as glioblastoma and lung adenocarcinoma $(16,17)$. In addition, some studies suggested that overexpression of CDK6 could lead to the acceleration of G1/S checkpoint in the cell cycle, which directly led to the enhancement of cell proliferation (18). This study found that CDK6 was highly expressed in thyroid cancer tissues and thyroid cancer cells, suggesting that CDK6 may also play an oncogenic part in thyroid cancer. Accordingly, CDK6 in SW579 and KHM-2 cells was regulated and observed. When CDK6 was inhibited, the proliferation of SW579 and KHM-2 cells was markedly suppressed, the apoptosis rate was significantly increased, and the detection of apoptosis-related proteins was consistent with the apoptotic rate. However, the phenotype observed in the further upregulated CDK6 was contrary, which confirmed our hypothesis. As stated in previous studies $(19,20)$, CDK6 activation first occurred in the middle of G1 phase, and could regulate the activity of $\mathrm{Rb}$ by phosphorylation. Some revealed that CDK6 regulated the cell growth and cell cycle progression mainly through transcriptional regulation, and that (21) CDK6 is a cofactor of NF- $\mathrm{KB}$, which could regulate cell cycle by interacting with NF- $\kappa B$ subunit $\mathrm{p} 65$. Furthermore, it was shown that CDK6 was overexpressed in non-small cell lung cancer, and that phosphorylation of CDK6 could lead to E2F-dependent transcription of essential cyclase and regulatory factors, as well as the assembly of prereplication complexes (22).

In conclusion, miR-298 can inhibit the proliferation of thyroid cancer cells and promote their apoptosis by inhibiting the expression of CDK6, which may be a new target for thyroid cancer therapy.

\section{Acknowledgements}

Not applicable.

\section{Funding}

This study was supported by Natural Science Project of CangzhouMedical College (18Z017).

\section{Availability of data and materials}

The datasets used and/or analyzed during the present study are available from the corresponding author on reasonable request.

\section{Authors' contributions}

$\mathrm{XL}$ wrote the manuscript. $\mathrm{CuL}$ and $\mathrm{XZ}$ conceived and designed the study. RW and NG were responsible for the collection and analysis of the experimental data. HS and XL interpreted the data and drafted the manuscript. $\mathrm{LW}$ and ChL performed the experiments and revised the manuscript critically for important intellectual content. All authors read and approved the final manuscript.

\section{Ethics approval and consent to participate}

The study was approved by the Ethics Committee of Cangzhou Medical College (Cangzhou, China). Patients who participated in this research had complete clinical data. Patients and their families agreed to participate in the experiment and signed informed consents were obtained from the patients or the guardians.

\section{Patient consent for publication}

Not applicable.

\section{Competing interests}

The authors declare that they have no competing interests.

\section{References}

1. Zhang T, He L, Sun W, Qin Y, Zhang P and Zhang H: 1,25-Dihydroxyvitamin D3 enhances the susceptibility of anaplastic thyroid cancer cells to adriamycin-induced apoptosis by increasing the generation of reactive oxygen species. Mol Med Rep 20: 2641-2648, 2019.

2. Luo Y,Hao T,Zhang J,Zhang M,Sun P and Wu L: MicroRNA-592 suppresses the malignant phenotypes of thyroid cancer by regulating lncRNA NEAT1 and downregulating NOVA1. Int J Mol Med 44: 1172-1182, 2019.

3. Bai J, Gao Y, Du Y, Yang X and Zhang X: MicroRNA-300 inhibits the growth of hepatocellular carcinoma cells by downregulating CREPT/Wnt/ $\beta$-catenin signaling. Oncol Lett 18: 3743-3753, 2019.

4. Zou L, Gao Z, Zeng F, Xiao J, Chen J, Feng X, Chen D, Fang Y, Cui J, Liu Y, et al: Sulfasalazine suppresses thyroid cancer cell proliferation and metastasis through T-cell originated protein kinase. Oncol Lett 18: 3517-3526, 2019.

5. Fuziwara CS, Saito KC and Kimura ET: Interplay of TGF $\beta$ signaling and microRNA in thyroid cell loss of differentiation and cancer progression. Arch Endocrinol Metab 63: 536-544, 2019.

6. Shi D, Wang H, Ding M, Yang M, Li C, Yang W and Chen L: MicroRNA-26a-5p inhibits proliferation, invasion and metastasis by repressing the expression of Wnt5a in papillary thyroid carcinoma. OncoTargets Ther 12: 6605-6616, 2019.

7. Jin J, Zhang J, Xue Y, Luo L, Wang S and Tian H: miRNA-15a regulates the proliferation and apoptosis of papillary thyroid carcinoma via regulating AKT pathway. OncoTargets Ther 12: 6217-6226, 2019.

8. Mo Y, He L, Lai Z, Wan Z, Chen Q, Pan S, Li L, Li D, Huang J, Xue F, et al: LINC01287/miR-298/STAT3 feedback loop regulates growth and the epithelial-to-mesenchymal transition phenotype in hepatocellular carcinoma cells. J Exp Clin Cancer Res 37: 149, 2018. 
9. Zhou F, Chen J and Wang H: MicroRNA-298 inhibits malignant phenotypes of epithelial ovarian cancer by regulating the expression of EZH2. Oncol Lett 12: 3926-3932, 2016.

10. Meyerson M and Harlow E: Identification of G1 kinase activity for cdk6, a novel cyclin D partner. Mol Cell Biol 14: 2077-2086, 1994.

11. Ferrari SM, Fallahi P, Galdiero MR, Ruffilli I, Elia G, Ragusa F, Paparo SR, Patrizio A, Mazzi V, Varricchi G, et al: Immune and inflammatory cells in thyroid cancer microenvironment. Int J Mol Sci 20: 20, 2019.

12. Liu W: lncRNA LINC-PINT inhibits cancer cell proliferation, invasion, and migration in osteosarcoma by downregulating miRNA-21. Cancer Biother Radiopharm 34: 258-263, 2019.

13. Hu S, Liao Y, Zheng J, Gou L, Regmi A, Zafar MI and Chen L: In silico integration approach reveals key microRNAs and their target genes in follicular thyroid carcinoma. BioMed Res Int 2019: 2725192, 2019.

14. Cao N, Mu L, Yang W, Liu L, Liang L and Zhang H: MicroRNA-298 represses hepatocellular carcinoma progression by inhibiting CTNND1-mediated Wnt/ $\beta$-catenin signaling. Biomed Pharmacother 106: 483-490, 2018.

15. Jin Y-P, Hu Y-P, Wu X-S, Wu YS, Ye YY, Li HF, Liu YC, Jiang L, Liu FT, Zhang YJ, et al: miR-143-3p targeting of ITGA6 suppresses tumour growth and angiogenesis by downregulating PLGF expression via the PI3K/AKT pathway in gallbladder carcinoma. Cell Death Dis 9: 182, 2018.

16. Lam PY, Di Tomaso E, Ng HK, Pang JC, Roussel MF and Hjelm NM: Expression of p19INK4d, CDK4, CDK6 in glioblastoma multiforme. Br J Neurosurg 14: 28-32, 2000.
17. Mendrzyk F, Radlwimmer B, Joos S, Kokocinski F, Benner A, Stange DE, Neben K, Fiegler H, Carter NP, Reifenberger G, et al: Genomic and protein expression profiling identifies CDK6 as novel independent prognostic marker in medulloblastoma. J Clin Oncol 23: 8853-8862, 2005.

18. Schwartz EI, Smilenov LB, Price MA, Osredkar T, Baker RA, Ghosh S, Shi FD, Vollmer TL, Lencinas A, Stearns DM, et al: Cell cycle activation in postmitotic neurons is essential for DNA repair. Cell cycle 6: 318-329, 2007.

19. Grossel MJ and Hinds PW: From cell cycle to differentiation: The role of cdk6 continues to expand. Cell Cycle 5: 266-270, 2006.

20. Handschick K, Beuerlein K, Jurida L, Bartkuhn M, Müller H, Soelch J, Weber A, Dittrich-Breiholz O, Schneider H, Scharfe M, et al: Cyclin-dependent kinase 6 is a chromatin-bound cofactor for NF- $\mathrm{kB}$-dependent gene expression. Mol Cell 53: 193-208, 2014.

21. Li B, He H, Tao BB, Zhao ZY, Hu GH, Luo C, Chen JX, Ding XH, Sheng P, Dong Y, et al: Knockdown of CDK6 enhances glioma sensitivity to chemotherapy. Oncol Rep 28: 909-914, 2012.

22. Ma H, Chen J, Pan S, Dai J, Jin G, Hu Z, Shen H and Shu Y: Potentially functional polymorphisms in cell cycle genes and the survival of non-small cell lung cancer in a Chinese population. Lung Cancer 73: 32-37, 2011.

This work is licensed under a Creative Commons Attribution-NonCommercial-NoDerivatives 4.0 International (CC BY-NC-ND 4.0) License. 